\title{
Evaluation of 5-Azacytidine as an inducer of osteoblastic / cementoblastic differentiation of progenitor cells from the periodontal ligament of humans
}

\section{Karoline de Moraes dos Santos*, Karina Gonzales Silvério Ruiz, Catharina Marques Sacramento.}

\begin{abstract}
Periodontal ligament (PDL) is constituted by heterogeneous cell populations with different cell differentiation capacities, here we investigated the effect of an epigenetic modulator on the induction of osteo/cementoblastic differentiation of human periodontal ligament mesenchymal progenitor cell clones with positive surface markers for CD105 (hPDLCD105+) and with low osteo/cementoblastic potential (LOP). These clones were pretreated with 5-aza-2'-deoxycytidine (5-aza-dC) and subjected to mineralization assays (Alizarin Red and Xylenol Orange), transcript levels' analysis of ALP, ANXA2, ASPN, BMP2, FGF7, OCN, OPN and RUNX2, using qRT-PCR. As results, 5-aza-dC enhances the capacity of mineralized matrix deposition of two LOP clones (L1 and L2), however different results regarding to the quantity of calcium nodules production was found, where L1 mineralization and osteogenic-related gene expression was greater than L2. The data show that the augmentation of BMP2, FGF7 and ANXA2 transcript levels may lead to a better osteo/cementogenic response, while the elevation of ASPN gene expression may block the mineralization performance of LOP clones. These results showed that pre-treatment with 5-aza-dC can positively modulate the osteo/cementogenic differentiation of hPDL-CD105+ cell clones with low osteo/ emento-blastic potential. Those findings contribute to better understand the complex mechanisms that can lead PDL stem cells to regenerate the periodontal tissues.
\end{abstract}

\section{Key words:}

Cell differentiation, gene expression, stem cells.

\section{Introduction}

Epigenetic modulators have been used to promote DNA changes and thus regulate signaling and gene expression involved in the differentiation of multipotent mesenchymal cells. Considering that a subset of hPDLSC are characterized as having low potential to form mineralized tissues, the aim of the present study was to investigate the role of ASPN, AXNA2, BMP2 and FGF7 markers on the regulation of osteoblast/cementoblast differentiation of these cell subset. Furthermore, we evaluated if osteo/cementoblastic potential of hPDLSCs can be modulated by epigenetic modifications of DNA.

\section{Results and Discussion}

(A)

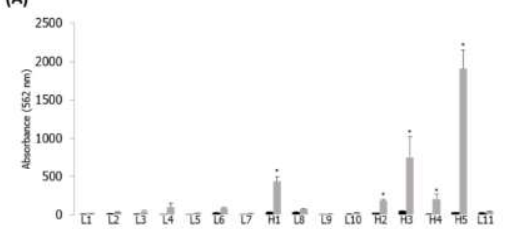

(B)

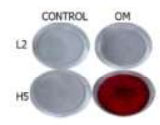

Image1. A) Quantification of Alizarin Red Staining (AR-S) of mineralized matrix deposition of sixteen PDL-CD105+ cell clones cultured in standard medium (CONTROL) and osteogenic medium (OM) for 28 days. A total of five clones were identified as high osteoblastic potential $(\mathrm{H} 1, \mathrm{H} 2, \mathrm{H} 3, \mathrm{H} 4$, and $\mathrm{H} 5)$, while eleven clones were characterized as low osteoblastic potential (L1, L2, L3, L4, L5, L6, L7, L8, L9, L10, and L11). B) AR-S for mineral nodule formation at $28 \mathrm{~d}$ after cell culture showed visual difference between LOP and HOP cell clones. L2 and H5 represent a LOP and HOP cell clones, respectively.

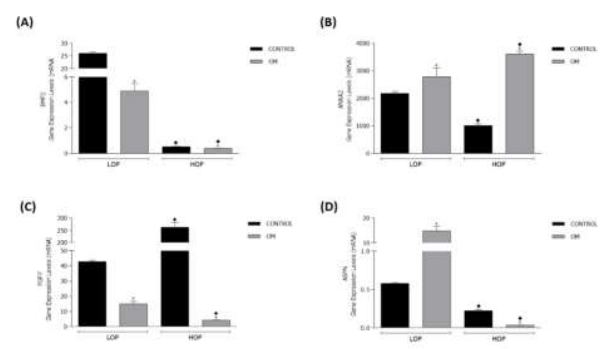

Image2. The modulation of BMP2, FGF7 and ANXA2 gene expression are similar in HOP and LOP cell clones after osteogenic induction but different for ASPN gene. qRT-PCR for BMP2 (A), ANXA2 (B), FGF7 (C) and ASPN (D) in LOP and HOP PDL-CD105+ cell clones cultured in control and osteogenic medium for 14 days.

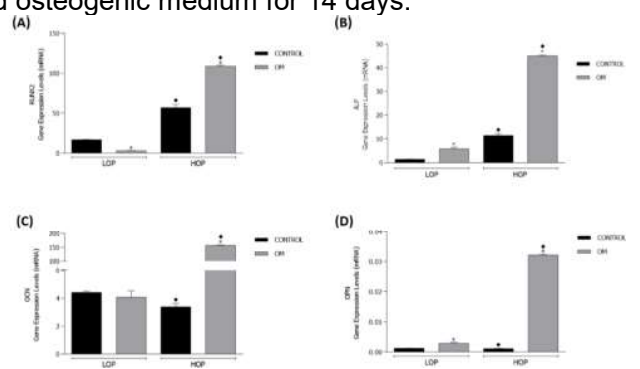

Image3. qRT-PCR for RUNX2 (A), ALP (B), OCN (C) and OPN (D) in low osteoblastic potential (LOP) and high osteoblastic potential (HOP) PDLCD105+ cell clones cultured in control and osteogenic medium for 14 days

\section{Conclusions}

In view of the results we can conclude that the pretreatment with 5 -aza-dC for 48 hours increases the capacity osteo / cementoblast analysis of human cell clones with surface markers positive for CD105 and that present low potential for differentiation osteo / cementoblastic. Although the capacity of mineralized matrix production was potentiated in the analyzed clones (L1 and L2), there was a difference in the amount of deposition of mineral nodules and gene expression of genes related to osteo / cementogenesis among these clones. In addition, the phenotype change promoted by 5aza-dC seems to be correlated with expression and modulation epigenetics of regulatory genes such as BMP2, ANXA2, FGF7 and ASPN. The results of this study will help us to understand the complex mechanisms that can lead to the stem cells present in the periodontal ligament to regenerate the tissues of support, improving future clinical approaches. 